\title{
A note on palindromic $\delta$-vectors for certain rational polytopes
}

\author{
Matthew H. J. Fiset and Alexander M. Kasprzyk* \\ Department of Mathematics and Statistics \\ University of New Brunswick \\ Fredericton, NB, Canada \\ u0a35@unb.ca, kasprzyk@unb.ca
}

Submitted: May 19, 2008; Accepted: Jun 1, 2008; Published: Jun 6, 2008

Mathematics Subject Classifications: 05A15, 11H06

\begin{abstract}
Let $P$ be a convex polytope containing the origin, whose dual is a lattice polytope. Hibi's Palindromic Theorem tells us that if $P$ is also a lattice polytope then the Ehrhart $\delta$-vector of $P$ is palindromic. Perhaps less well-known is that a similar result holds when $P$ is rational. We present an elementary lattice-point proof of this fact.
\end{abstract}

\section{Introduction}

A rational polytope $P \subset \mathbb{R}^{n}$ is the convex hull of finitely many points in $\mathbb{Q}^{n}$. We shall assume that $P$ is of maximum dimension, so that $\operatorname{dim} P=n$. Throughout let $k$ denote the smallest positive integer for which the dilation $k P$ of $P$ is a lattice polytope (i.e. the vertices of $k P$ lie in $\mathbb{Z}^{n}$ ).

A quasi-polynomial is a function defined on $\mathbb{Z}$ of the form:

$$
q(m)=c_{n}(m) m^{n}+c_{n-1}(m) m^{n-1}+\ldots+c_{0}(m),
$$

where the $c_{i}$ are periodic coefficient functions in $m$. It is known ([Ehr62] that for a rational polytope $P$, the number of lattice points in $m P$, where $m \in \mathbb{Z}_{\geq 0}$, is given by a quasi-polynomial of degree $n=\operatorname{dim} P$ called the Ehrhart quasi-polynomial; we denote this by $L_{P}(m):=\left|m P \cap \mathbb{Z}^{n}\right|$. The minimum period common to the cyclic coefficients $c_{i}$ of $L_{P}$ divides $k$ (for further details see [BSW08]).

${ }^{*}$ The first author was funded by an NSERC USRA grant. The second author is funded by an ACEnet research fellowship. 
Stanley proved in [Sta80] that the generating function for $L_{P}$ can be written as a rational function:

$$
\operatorname{Ehr}_{P}(t):=\sum_{m \geq 0} L_{P}(m) t^{m}=\frac{\delta_{0}+\delta_{1} t+\ldots+\delta_{k(n+1)-1} t^{k(n+1)-1}}{\left(1-t^{k}\right)^{n+1}}
$$

whose coefficients $\delta_{i}$ are non-negative. For an elementary proof of this and other relevant results, see [BS07] and [BR07]. We call $\left(\delta_{0}, \delta_{1}, \ldots, \delta_{k(n+1)-1}\right)$ the (Ehrhart) $\delta$-vector of $P$.

The dual polyhedron of $P$ is given by $P^{\vee}:=\left\{u \in \mathbb{R}^{n} \mid\langle u, v\rangle \leq 1\right.$ for all $\left.v \in P\right\}$. If the origin lies in the interior of $P$ then $P^{\vee}$ is a rational polytope containing the origin, and $P=\left(P^{\vee}\right)^{\vee}$. We restrict our attention to those $P$ containing the origin for which $P^{\vee}$ is a lattice polytope.

We give an elementary lattice-point proof that, with the above restriction, the $\delta$ vector is palindromic (i.e. $\delta_{i}=\delta_{k(n+1)-1-i}$ ). When $P$ is reflexive, meaning that $P$ is also a lattice polytope (equivalently, $k=1$ ), this result is known as Hibi's Palindromic Theorem Hib91]. It can be regarded as a consequence of a theorem of Stanley's concerning the more general theory of Gorenstein rings; see [Sta78].

\section{The main result}

Let $P$ be a rational polytope and consider the Ehrhart quasi-polynomial $L_{P}$. There exist $k$ polynomials $L_{P, r}$ of degree $n$ in $l$ such that when $m=l k+r$ (where $l, r \in \mathbb{Z}_{\geq 0}$ and $0 \leq r<k$ ) we have that $L_{P}(m)=L_{P, r}(l)$. The generating function for each $L_{P, r}$ is given by:

$$
\operatorname{Ehr}_{P, r}(t):=\sum_{l \geq 0} L_{P, r}(l) t^{l}=\frac{\delta_{0, r}+\delta_{1, r} t+\ldots+\delta_{n, r} t^{n}}{(1-t)^{n+1}}
$$

for some $\delta_{i, r} \in \mathbb{Z}$.

Theorem 2.1. Let $P$ be a rational n-tope containing the origin, whose dual $P^{\vee}$ is a lattice polytope. Let $k$ be the smallest positive integer such that $k P$ is a lattice polytope. Then:

$$
\delta_{i, r}=\delta_{n-i, k-r-1}
$$

Proof. By Ehrhart-Macdonald reciprocity ([Ehr67, Mac71]) we have that:

$$
L_{P}(-l k-r)=(-1)^{n} L_{P^{\circ}}(l k+r),
$$

where $L_{P}$ o enumerates lattice points in the strict interior of dilations of $P$. The lefthand side equals $L_{P}(-(l+1) k+(k-r))=L_{P, k-r}(-(l+1))$. We shall show that the right-hand side is equal to $(-1)^{n} L_{P}(l k+r-1)=(-1)^{n} L_{P, r-1}(l)$.

Let $H_{u}:=\left\{v \in \mathbb{R}^{n} \mid\langle u, v\rangle=1\right\}$ be a bounding hyperplane of $P$, where $u \in \operatorname{vert} P^{\vee}$. By assumption, $u \in \mathbb{Z}^{n}$ and so the lattice points in $\mathbb{Z}^{n}$ lie at integer heights relative to 
$H_{u}$; i.e. given $u^{\prime} \in \mathbb{Z}^{n}$ there exists some $c \in \mathbb{Z}$ such that $u^{\prime} \in\left\{v \in \mathbb{R}^{n} \mid\langle u, v\rangle=c\right\}$. In particular, there do not exist lattice points at non-integral heights. Since:

$$
P=\bigcap_{u \in \operatorname{vert} P^{\vee}} H_{u}^{-}
$$

where $H_{u}^{-}$is the half-space defined by $H_{u}$ and the origin, we see that $\left(m P^{\circ}\right) \cap \mathbb{Z}^{n}=$ $((m-1) P) \cap \mathbb{Z}^{n}$. This gives us the desired equality.

We have that $L_{P, k-r}(-(l+1))=(-1)^{n} L_{P, r-1}(l)$. By considering the expansion of (2.1) we obtain:

$$
\begin{aligned}
\sum_{i=0}^{n} \delta_{i, k-r}\left(\begin{array}{c}
-(l+1)+n-i \\
n
\end{array}\right) & =L_{P, k-r}(-(l+1)) \\
& =(-1)^{n} L_{P, r-1}(l)=(-1)^{n} \sum_{i=0}^{n} \delta_{i, r-1}\left(\begin{array}{c}
l+n-i \\
n
\end{array}\right) .
\end{aligned}
$$

But $\left(\begin{array}{c}-(l+1)+n-i \\ n\end{array}\right)=(-1)^{n}\left(\begin{array}{c}l+n-i \\ n\end{array}\right)$, and since $\left(\begin{array}{c}l \\ n\end{array}\right),\left(\begin{array}{c}l+1 \\ n\end{array}\right), \ldots,\left(\begin{array}{c}l+n \\ n\end{array}\right)$ form a basis for the vector space of polynomials in $l$ of degree at most $n$, we have that $\delta_{i, k-r}=\delta_{n-i, r-1}$.

Corollary 2.2. The $\delta$-vector of $P$ is palindromic.

Proof. This is immediate once we observe that:

$$
\operatorname{Ehr}_{P}(t)=\operatorname{Ehr}_{P, 0}\left(t^{k}\right)+t \operatorname{Ehr}_{P, 1}\left(t^{k}\right)+\ldots+t^{k-1} \operatorname{Ehr}_{P, k-1}\left(t^{k}\right)
$$

\section{Concluding remarks}

The crucial observation in the proof of Theorem 2.1 is that $\left(m P^{\circ}\right) \cap \mathbb{Z}^{n}=((m-1) P) \cap \mathbb{Z}^{n}$. In fact, a consequence of Ehrhart-Macdonald reciprocity and a result of Hibi Hib92 tells us that this property holds if and only if $P^{\vee}$ is a lattice polytope. Hence rational convex polytopes whose duals are lattice polytopes are characterised by having palindromic $\delta$ vectors. This can also be derived from Stanley's work [Sta78] on Gorenstein rings.

\section{References}

[BR07] Matthias Beck and Sinai Robins, Computing the continuous discretely, Undergraduate Texts in Mathematics, Springer, New York, 2007, Integer-point enumeration in polyhedra.

[BS07] Matthias Beck and Frank Sottile, Irrational proofs for three theorems of Stanley, European J. Combin. 28 (2007), no. 1, 403-409. 
[BSW08] Matthias Beck, Steven V. Sam, and Kevin M. Woods, Maximal periods of (Ehrhart) quasi-polynomials, J. Combin. Theory Ser. A 115 (2008), no. 3, 517525 .

[Ehr62] Eugène Ehrhart, Sur les polyèdres homothétiques bordés à n dimensions, C. R. Acad. Sci. Paris 254 (1962), 988-990.

[Ehr67] _ Sur un problème de géométrie diophantienne linéaire. II. Systèmes diophantiens linéaires, J. Reine Angew. Math. 227 (1967), 25-49.

[Hib91] Takayuki Hibi, Ehrhart polynomials of convex polytopes, h-vectors of simplicial complexes, and nonsingular projective toric varieties, Discrete and computational geometry (New Brunswick, NJ, 1989/1990), DIMACS Ser. Discrete Math. Theoret. Comput. Sci., vol. 6, Amer. Math. Soc., Providence, RI, 1991, pp. $165-177$.

[Hib92] _ Dual polytopes of rational convex polytopes, Combinatorica 12 (1992), no. 2, 237-240.

[Mac71] I. G. Macdonald, Polynomials associated with finite cell-complexes, J. London Math. Soc. (2) 4 (1971), 181-192.

[Sta78] Richard P. Stanley, Hilbert functions of graded algebras, Advances in Math. 28 (1978), no. 1, 57-83.

[Sta80] - Decompositions of rational convex polytopes, Ann. Discrete Math. 6 (1980), 333-342, Combinatorial mathematics, optimal designs and their applications (Proc. Sympos. Combin. Math. and Optimal Design, Colorado State Univ., Fort Collins, Colo., 1978). 